\title{
Allopurinol desensitization with A 2 weeks modified protocol in an elderly patients with multiple comorbidities: a case report
}

\author{
Adile Berna Dursun ${ }^{1 *}$ and Osman Z Sahin ${ }^{2}$
}

\begin{abstract}
Background: Allopurinol is an effective urate-lowering drug that is well tolerated by the majority of patients. Patients with chronic renal insufficiency have an increased risk of hypersensitivity reactions with allopurinol.

Case presentation: 75 year old male patient with gout, renal insufficiency, history of metastatic colorectal carcinoma status post-resection was referred to Allergy clinic for a maculopapular eruption that developed 1 week after initiating therapy with allopurinol. The rash resolved with discontinuation of allopurinol. However, his serum urate level rose to $19.9 \mathrm{mg} / \mathrm{dl}$. We initially proposed a slow 4 week oral allopurinol desensitization. The treating nephrologist felt it was critical to lower urate more rapidly. As a result, we modified the dose and standard 4 week protocol down to 2 weeks. A suspension of allopurinol was prepared by the allergy nurse practitioner with a $300 \mathrm{mg}$ allopurinol tablet. The sensitization protocol was modified as a starting dose of $0.3 \mathrm{mg}$ escalating to a final dose of $300 \mathrm{mg} /$ day in 2 weeks. There was no reaction during or after the desensitization. The patient's urate level normalized $(6.3 \mathrm{mg} / \mathrm{dl})$ and has continued on $300 \mathrm{mg}$ allopurinol daily without reaction.
\end{abstract}

Conclusion: A 2 week modified allopurinol desensitization protocol is a safe alternative for elderly patients with multiple comorbidities.

Keywords: Allopurinol hypersensitivty, Oral drug desensitization, Slow desensitization, Hyperuricemia, Gout, Maculopapular exanthema

Allopurinol is a first line drug for treatment of gout and works by inhibition of xanthine oxidase leading to decreased uric acid produciton [1]. Although it is a welltolerated drug, approximately $2 \%$ of patients treated with allopurinol have adverse reactions such as fixed drug eruptions (FDE), pruritic maculopapular exanthema (MPE) and minor vasculitis whicch resolve with cessation of the drug which limits it use in these individuals [2]. Life-threatening reactions such as DRESS; SJS or TEN, occur very infrequently $(0.2 \%)[3,4]$.

Approach to allopurinol hypersensitivity firstly includes withdrawing allopurinol, giving supportive measures such as modifying diet and alcohol intake and corticosteroids in severe cases [3]. The other uricosuric

\footnotetext{
* Correspondence: aberna.dursun@gmail.com

${ }^{1}$ Department of Internal Medicine, Division of Immunology and Allergic Diseases, Recep Tayyip Erdogan University, School of Medicine, Rize, Turkey Full list of author information is available at the end of the article
}

drugs (probenicid, benzbromarone and sulfinpyrazone) are another option in the chronic treatment of gout [5]. When allopurinol treatment is necessary and there is no alternative drugs in the local market, drug desensitization should be considered except for severe hypersentivity reactions [4].

After first publication of desensitization with allopurinol in 1976, several case reports with oral and intravenous protocols were published $[5,6]$. Slow drug desensitization protocol lasting in 28-81 days are recommended particularly for elderly patients with multiple comorbidities [2]. We present a successful modified 2 weeks oral allopurinol desensitization in an elderly patient with multiple comorbidities.

\section{Case presentation}

A 75 years old male was consulted due to hypersensitivty reaction to allopurinol. He had chronic renal disease, metastatic colorectal carcinoma status post-resection. 
Neither the patient nor his family members had a history of drug hypersensitivity. A week after starting allopurinol (300 mg/day), he presented to dermatology out-patient clinic with rashes on his lower extremities. He was diagnosed with allopurinol-induced MPE. Allopurinol was discontinued and he was placed on 5 days of oral corticosteroids. His rash resolved however, his serum urate level rose to $19.9 \mathrm{mg} / \mathrm{dl}$ (normal range $3-7.5 \mathrm{mg} / \mathrm{dl}$ ) and he experienced a gout attack. There were no alternative urate lowering drugs in our market. Febuxostat could be obtained with board report from Ministry of Health but would take at least 3 weeks.

Due to his critic condition, a 28-days allopurinol desensitization protocol was modified to 2 weeks and was performed without premedication (Table 1). The protocol was modified from a protocol developed by Gomes et al. [5]. The soluble suspension of allopurinol was prepared by allergy nurse practitioner as described in Table 2. Solutions were well tolerated by the patient without complaints. The patient was seen at the out-patient clinic on $1^{\text {st }}, 6 \mathrm{t}^{\mathrm{h}}, 9^{\text {th }}, 12^{\text {th }}$ and $16^{\text {th }}$ days of the protocol. No reaction occured during the desensitization or during continued daily dosing of allopurinol $300 \mathrm{mg}$. The uric acid level dropped to $6.3 \mathrm{mg} / \mathrm{dl}$ with therapy.

\section{Discussion}

Although allopurinol hypersensitivity is relatively uncommon, up to $2 \%$ of patients who use this drug develop hypersensitivity reactions such as FDE, pruritic MPE and vasculitis [2]. Despite the fact that new drugs have been developed to treat hyperuricemia, practical therapeutic

\section{Table 12 weeks allopurinol desensitization protocol}

\begin{tabular}{cccc}
\hline DAY & DRUG (Allopurinol) & DOSE $(\mathbf{m l})$ & DOSE $(\mathbf{m g})$ \\
\hline 1 & Solution A, 0.3 mg/ml & 1 & 0.3 \\
2 & 2 & 0.6 \\
3 & 4 & 1.2 \\
4 & 8 & 2.4 \\
5 & Solution B, 6 mg/ml & 10 & 3 \\
6 & 3 & 18 \\
7 & & 6 & 36 \\
8 & & 10 & 60 \\
9 & Allopurinol tablet (100-300 mg) & 75 \\
10 & & & 100 \\
11 & & 125 \\
12 & & 150 \\
13 & & 175 \\
14 & & 225 \\
15 & & 250 \\
16 & & 300 \\
\hline
\end{tabular}

Table 2 Description of preparing allopurinol for desensitization

\begin{tabular}{ll}
\hline Source solution & $\begin{array}{l}150 \mathrm{mg} \text { allopurinol tablet }+50 \mathrm{ml} \mathrm{5 \%} \\
\text { dextrose }=3 \mathrm{mg} / \mathrm{ml}\end{array}$ \\
Solution A & $1: 10$ source solution $(0.3 \mathrm{mg} / \mathrm{ml})$ \\
Solution B & $300 \mathrm{mg}$ allopurinol tablet $+50 \mathrm{ml} 5 \%$ \\
& dextrose $=6 \mathrm{mg} / \mathrm{ml}$
\end{tabular}

choices remain limited. Febuxostat is a good option for patients with allopurinol hypersensitivity, but its accessibility may be very limited in some countries $[2,4]$. In this situation, desensitization procedure should be considered, except for patients with severe hypersensitivity to allopurinol. FDE and maculopapular exanthema is an appropriate indication for desensitization with allopurinol [5].

The pathophysiology underlying this hypersensitivity reaction remains unknown, but several risk factors such as the presence of the HLA-B58.01 allele, the dose taken and renal failure have been proposed. Recently Yun et al. found that allopurinol allergic patients are primarily sensitized to oxypurinol. Despite the prevailing dogma that type B adverse drug reactions are dose independent, they concluded that allopurinol hypersensitivity is primarily driven by oxypurinol-specific $\mathrm{T}$ cell response in a dosedependent manner, particular in the presence of HLAB58.01 allele [7].

Different mechanisms, including hapten inhibition, mast cell and basophil mediator depletion, IgE consumption and mast cell desensitization, have also been proposed to explain the temporary immunological tolerance induced by a desensitization protocol to a drug. The mechanism of slow desensitization is more obscure. It has been suggested that gradually increasing antigen doses allows metabolic adaptation resulting in increased clearance of reactive drug metabolites [2].

There are several oral slow desensitization protocols lasted in 28 to 81 days in the literature [5,8-10]. Time to reach therapeutic doses is longer particularly in elderly patients with multiple comorbidities for safety issues [2]. However if there is clinical urgency, new or modified protocols could be tailored. The original protocol was generated by Umperierrez et al. [9] for a patient with FDE to allopurinol, then Gomes et al. adapted the protocol for also systemic reactions to allopurinol [5]. In the Gomes study, the type of reaction was FDE in three patients, urticarial with or without angioedema in two, anaphylaxis in one and pruritic MPE in one patient. They reported $100 \%$ overall success rate of desensitization with no complications in the progression of the protocol in three patients (43\%) and the remaining had mild to moderate skin reactions [5]. Even though the protocol was initially created for desensitization of 
patients with local skin reactions, Gomes et al. confirmed that it is also effective in desensitization of patients with systemic reactions [5]. Thus we also modified the same protocol for patient with MPE to allopurinol and the successful outcome revealed the flexibility of slow desensitization protocols.

It is important to share new or modified drug desensitization protocols, whether they have success or not, in order to build up experiences in a field of insufficient data [11]. We present a successful shortened oral desensitization protocol for allopurinol hypersensitivity. The presented case demonstrated that a 2 weeks oral allopurinol desensitization protocol can be safely undertaken in elderly patients with multiple comorbidities.

\section{Consent}

Written informed consent was obtained from the patient for publication of this case report and any accompanying images. A copy of written inform consent is available for review by the Editor-in-Chief of this journal.

\section{Competing interests}

The authors declare that they have no competing interests.

\section{Authors' contributions}

$\mathrm{OZS}, \mathrm{ABD}$ acquisition of data, anaylsis and interpretation of data and drafting manuscript. ABD Critical revision. Both authors read and approved the final manuscript.

\section{Author details}

'Department of Internal Medicine, Division of Immunology and Allergic Diseases, Recep Tayyip Erdogan University, School of Medicine, Rize, Turkey. ${ }^{2}$ Department of Internal Medicine, Division of Nephrology, Recep Tayyip Erdogan University, School of Medicine, Rize, Turkey.

Received: 13 August 2014 Accepted: 8 October 2014

Published: 22 October 2014

\section{References}

1. Pacher P, Nivorozhkin A, Szabo C: Therapeutic effects of xanthine oxidase inhibitors: renaissance half century after discovery of allopurinol. Pharmacol Rev 2006, 58:87-114.

2. Calogiuri G, Nettis E, Di Leo E, Foti C, Ferrannini A, Butani L: Allopurinol hypersensitivity reactions: desensitization strategies and new therapeutic alternative molecules. Inflamm Allergy-Drug Targets 2013, 12:19-28.

3. Gutierrez-Macias A, Lizarralde-Palacios E, Martinez-Odriozola P, Miguel-De la Villa F: Fatal allopurinol hypersensitivity syndrome after treatment of asymptomatic hyperuricemia. BMJ 2005, 331:623-624.

4. Monev SD: How should hyperuricemia be treated in a patient with allopurinol hypersensitivity? Cleveland Clin J Med 2001, 68(7):597-598.

5. Gomes R, Ribeiro F, Faria E, Loureiro C, Segorbe-Luis A: Desensitization to allopurinol in localized and systemic hypersensitivity reactions. J Allergy Ther 2013, 4(4):1000138.

6. Meyrier A: Desensitization in a patient with chronic renal disease and severe allergy to allopurinol. Br Med J 1976, 2:458.

7. Yun J, Mattsson J, Schnyder K, Fontana S, Largiader CR, Pichler WJ, Yerly D: Allopurinol hypersensitivity is primarily mediated by dose-dependent oxypurinol-specific T cell response. Clin Exp Allergy 2013, 438(11):1246-1255

8. Kelso JM, Keating RM: Successful desensitization for treatment of a fixed drug eruption to allopurinol. J Allergy Clin Immunol 1996, 97:1171-1172.
9. Umperierrez A, Herrranz JC, De Las Heras M, Lluch-Bernal M, Figueredo E, Sastre J: Successful desensitization of a fixed drug eruption caused by allopurinol. J Allergy Clin Immunol 1998, 101:286-287.

10. Fam AG, Dunne SM, lazette J, Paton TW: Efficacy and safety of desensitization to allopurinol following cutaneous reactions. Arthritis Rheum 2001, 44:231-238.

11. Chiriac AM, Demoly P: Drug provocation tests: up-date and novel approaches. Allergy Asthma Clin Immunol 2013, 9(1):12.

doi:10.1186/1710-1492-10-52

Cite this article as: Dursun and Sahin: Allopurinol desensitization with A 2 weeks modified protocol in an elderly patients with multiple comorbidities: a case report. Allergy, Asthma \& Clinical Immunology 2014 10:52.

\section{Submit your next manuscript to BioMed Central and take full advantage of:}

- Convenient online submission

- Thorough peer review

- No space constraints or color figure charges

- Immediate publication on acceptance

- Inclusion in PubMed, CAS, Scopus and Google Scholar

- Research which is freely available for redistribution 